\title{
Exact Capacities and Star-Shaped Distorted Probabilities
}

\author{
Zaier Aouani*, Alain Chateauneuf ${ }^{\dagger}$ \\ February 17, 2006
}

\begin{abstract}
In this work, we are interested in capacities which are deformations of probability i.e $v=f \circ P$. We characterize balanced, totally balanced, exact and convex capacities, by properties concerning the probability transformation function, $f$. We also give the explicit expression, in the case of a convex capacity $v=f \circ P$, of a probability in the core of $v$ which coincides with $v$ on a given finite chain of elements of the algebra $\mathcal{A}$. As a dividend of our analysis, we obtain simple new characterizations of a large pattern of risk aversions, relevant to the Yaari rank-dependent expected utility model.
\end{abstract}

Keywords: Capacity; exact; balanced; star-shaped function; risk aversion JEL Classification: C71, D80.

\section{Introduction}

Set functions, which are not necessarily additive, are frequently used in decision theory. Indeed, they have different interpretations: on the one hand, they represent transferable utility or the characteristic function of a cooperative game; while on the other hand, they represent non-additive probabilities.

In this work, we are interested in capacities which are deformations of a given probability, i.e.: $v=f \circ P$ where $P$ is a non-atomic additive probability on a measurable space $(\Omega, \mathcal{A})$ where $\Omega$ is a non-empty set, $\mathcal{A}$ a $\sigma$-algebra of subsets of $\Omega$ and $f:[0,1] \rightarrow[0,1]$ is a nondecreasing function, satisfying $f(0)=0$ and $f(1)=1$.

The main purpose of this paper is to characterize a wide classical range of monotone normalized games i.e capacities, by properties concerning the probability transformation $f$. As a dividend, building upon [3], this allows us to obtain directly simple new characterizations of a large pattern of risk attitudes relevant to Yaari's dual theory of choice under risk [14], in terms of simple specific properties of the capacity arising in Yaari's model.

\footnotetext{
${ }^{*}$ University of Kansas, Economics Department, 213 Summerfield Hall, Lawrence, KS 66045, USA; Aouani@ku.edu

${ }^{\dagger}$ Université Paris I, CERMSEM, UMR CNRS 8095, 106-112 boulevard de l'Hopital, 75647 Paris Cedex 13, France; Alain.Chateauneuf@univ-paris1.fr
} 
The paper is organized as follows: in the second section, we introduce some notations, definitions and preliminary results. Section 3 is essentially devoted to the characterization of balanced, totally balanced, exact and convex capacities by properties related to $f$. The most salient result, related to Wallner [13] who dealt with robust statistics, concerns exact capacities which are proved to be obtained through star-shaped distortions. In section 4 , results of section 3 are straightforwardly applied for deriving new characterizations of several kinds of risk aversion for decision makers à la Yaari. Section 5 concludes.

\section{Definitions, Notation and Preliminary Results}

A game is a triple $(\Omega, \mathcal{A}, v)$ where $\Omega$ is a non empty set, $\mathcal{A}$ is an algebra of subsets of $\Omega$ and $v$ is a function $v: \mathcal{A} \rightarrow \mathbb{R}_{+}$such that $v(\emptyset)=0$. If there is no risk of confusion we will speak simply about game $v$. The game $v$ is called a (normalized) capacity if (i) $v$ is normalized i.e. $v(\Omega)=1$, and (ii) $v$ is monotone i.e. $A, B \in \mathcal{A}$ and $A \subset B$ implies $v(A) \leqslant v(B)$. Each (normalized) capacity $v$ has a dual (normalized) capacity $\bar{v}$ defined by $\bar{v}(A)=1-v(\bar{A})$ for all $A \in \mathcal{A}$. Elements of $\Omega$ are usually called players (or agents) or states of nature and the elements of $\mathcal{A}$ are called coalitions or events, depending on whether the context is game theory or decision under uncertainty. $v(A)$ is interpreted then as a measurement of the power of coalition $A$ (for example in the division of a perfectly divisible good, $v(A)$ is the quantity of this good the agents of $A$ can obtain without the assistance of agents of the complementary coalition) or the "subjective probability" of the event $A$.

It is easy to check that if $\mathcal{A}$ is an algebra of subsets of $\Omega$ and $A \in \mathcal{A}$, the family $\mathcal{A} \cap A$ is an algebra of subsets of $A$. If $A \in \mathcal{A}$, then the game $\left(A, \mathcal{A} \cap A, v_{\mid \mathcal{A} \cap A}\right)$ is called the restriction of $v$ to the coalition $A$. The game $v_{\mid \mathcal{A} \cap A}$ will be simply denoted $v_{\mid A}$.

The core of a game $(\Omega, \mathcal{A}, v)$ is the set

$$
\operatorname{core}(v)=\left\{\lambda: \mathcal{A} \rightarrow \mathbb{R}_{+} \mid \lambda \text { is a finitely additive measure, } \lambda(\Omega)=v(\Omega) \text { and } \lambda \geqslant v\right\} .
$$

The core represents the set of possible divisions of the benefit $v(\Omega)$ where no coalition can protest in the name of its own capacity. It is also the set of probabilities which are considered by the decision maker, as susceptible to manage the situation of uncertainty.

A game $v$ is said to be:

(a) balanced if core $(v) \neq \emptyset$.

(b) totally balanced if for all $A \in \mathcal{A}$, its restriction to $A$ is balanced.

(c) exact if $\forall A \in \mathcal{A} ; v(A)=\min \{\mu(A) \mid \mu \in \operatorname{core}(v)\}$. The minimum in the preceeding expression is reached since core $(v)$ is compact for the weak- $\star$ topology of the space of additive and bounded measures.

(d) convex if $A_{1}$ and $A_{2} \in \mathcal{A}$ implies $v\left(A_{1}\right)+v\left(A_{2}\right) \leqslant v\left(A_{1} \cup A_{2}\right)+v\left(A_{1} \cap A_{2}\right)$. $v$ is called super-additive if the preceeding inequality holds whenever $A_{1}, A_{2} \in \mathcal{A}$ and $A_{1} \cap A_{2}=\emptyset$. 
An equivalent condition (due to Shapley [12]) to the convexity of $v$ is

$$
v\left(A_{1} \cup B\right)-v\left(A_{1}\right) \leqslant v\left(A_{2} \cup B\right)-v\left(A_{2}\right) ; \forall A_{1} \subseteq A_{2}, \forall B \text { with } A_{2} \cap B=\emptyset
$$

thus when $\Omega$ is finite:

$$
v\left(A_{1} \cup\{i\}\right)-v\left(A_{1}\right) \leqslant v\left(A_{2} \cup\{i\}\right)-v\left(A_{2}\right) ; \forall i \in \Omega, \forall A_{1} \subseteq A_{2} \subseteq \Omega \backslash\{i\} .
$$

This expresses an increasing marginal utility with certainty (in situation of decision under uncertainty) or with the number of members of a coalition (in game theory). Intuitively, it says that the "incentives" to join a coalition increase with its size (creating a "snowballing" effect), which is the analogue of the increasing outputs scales associated to a convex production function in an economy. Shapley [12] gave an elegant justification of the term "convex": let $B \in \mathcal{A}$, define a "differential" operator by: $\left[\Delta_{B} v\right](A)=v(A \cup$ $B)-v(A \backslash B) ; \forall A \in \mathcal{A}$. Let $\Delta_{C B} v=\Delta_{C}\left(\Delta_{B} v\right)$. Then the convexity of $v$ is equivalent to $\left[\Delta_{C B} v\right](A) \geqslant 0 ; \forall A, B, C \in \mathcal{A}$, which is similar to the positivity of second derivatives of a convex function in real analysis.

It is well-known (see for example [10]) that one has the following implications:

$$
v \text { is convex } \Rightarrow v \text { is exact } \Rightarrow v \text { is totally balanced } \Rightarrow v \text { is balanced. }
$$

We recall here characterizations of exact, balanced, totally balanced and convex games. To prove theorem 2.1, Kannai [9] used a fundamental result due to Ky Fan [6] concerning linear systems of inequalities in a real normed vector space. Schmeidler [10] proved the same result using a separation theorem. See also Chateauneuf [1].

For $A \subseteq \Omega, A^{*}$ denotes the characteristic function of $A$ i.e. the function defined on $\Omega$, being equal to 1 on $A$ and 0 elsewhere.

Theorem 2.1. Let $\mathcal{A}$ be a $\sigma$-algebra of subsets of $\Omega$ and $v$ a game. $v$ is exact iff for all $n \in \mathbb{N}^{*} ; a \in \mathbb{R} ; a_{1}, \ldots, a_{n} \in \mathbb{R}_{+} ; A, A_{1}, \ldots, A_{n} \in \mathcal{A}$ :

$$
A^{*}+a \Omega^{*} \geqslant \sum_{i=1}^{n} a_{i} A_{i}^{*} \Rightarrow v(A)+a v(\Omega) \geqslant \sum_{i=1}^{n} a_{i} v\left(A_{i}\right) .
$$

Corollary 2.1. The game $v$ is balanced if and only if $\forall n \in \mathbb{N}^{*} ; a_{1}, \ldots, a_{n} \geqslant 0$ and $A_{1}, \ldots, A_{n}$ $\in \mathcal{A}$ we have: $\sum_{i=1}^{n} a_{i} A_{i}^{*} \leqslant \Omega^{*} \Longrightarrow \sum_{i=1}^{n} a_{i} v\left(A_{i}\right) \leqslant v(\Omega)$.

Corollary 2.2. The game $v$ is totally balanced if and only if $\forall n \in \mathbb{N}^{*} ; \forall a_{1}, \ldots, a_{n} \geqslant$ 0 and $A, A_{1}, \ldots, A_{n} \in \mathcal{A}$ we have: $\sum_{i=1}^{n} a_{i} A_{i}^{*} \leqslant A^{*} \Longrightarrow \sum_{i=1}^{n} a_{i} v\left(A_{i}\right) \leqslant v(A)$.

Theorem 2.2. The game $v$ is convex if and only if for any finite sequence $\emptyset=A_{0} \subseteq A_{1} \subseteq$ $\ldots \subseteq A_{n}=\Omega$ in $\mathcal{A} ; \exists \lambda \in \operatorname{core}(v)$ such that $\lambda\left(A_{0}\right)=v\left(A_{0}\right), \ldots, \lambda\left(A_{n}\right)=v\left(A_{n}\right)$.

Proof. See [5]. 
We give in proposition 3.5. below, when $v$ is a convex distorted probability, an explicit expression of such a probability $\lambda$.

Recall the following definitions, introduced by Gilboa and Lehrer [8], which will be useful for the sequel. Anti $-\operatorname{core}(v)=\left\{\lambda: \mathcal{A} \rightarrow \mathbb{R}_{+}\right.$such that: $\lambda$ is a finitely additive measure, $\lambda(\Omega)=v(\Omega)$ and $\lambda \leqslant v\}$. Anti-balancedness and total anti-balancedness are then defined in a classic manner: $v$ is said to be anti-balanced if anti-core $(v) \neq \emptyset$. It is said to be totally anti-balanced if $\forall A \in \mathcal{A}, v_{\mid A}$ is anti-balanced. We have the following characterization that we prove for sake of completeness.

Proposition 2.1. $v$ is anti-balanced iff $\forall n \in \mathbb{N} ; \forall a_{1}, \ldots, a_{n} \geqslant 0$ and $A_{1}, \ldots, A_{n} \in \mathcal{A}$, the following implication holds: $\sum_{i=1}^{n} a_{i} A_{i}^{*} \geqslant \Omega^{*} \Rightarrow \sum_{i=1}^{n} a_{i} v\left(A_{i}\right) \geqslant v(\Omega)$.

Proof. $\Rightarrow$ : Immediate.

$\Leftarrow$ : We use Ky Fan's theorem [6]: Let $\left\{x_{\nu}\right\}_{\nu \in J}$ be a family of elements, not all 0 , in a real normed vector space $E$, and $\left\{\alpha_{\nu}\right\}_{\nu \in J}$ a family of corresponding real numbers. Let $\sigma=\sup \left\{\sum_{j=1}^{n} \beta_{j} \alpha_{\nu_{j}} \mid n \in \mathbb{N}^{*} ; \beta_{1}, \ldots, \beta_{n} \in \mathbb{R}_{+}^{*} ;\left\|\sum_{j=1}^{n} \beta_{j} x_{\nu_{j}}\right\|=1\right\}$. Then the system $f\left(x_{\nu}\right) \geqslant \alpha_{\nu} ; \forall \nu \in J$, has a solution $f \in E^{\prime}$ (the conjugate space of $E$ ) iff $\sigma$ is finite.

The question is then equivalent to finding $g \in \mathcal{V}^{\prime}$ (where $\mathcal{V}=\{X: \Omega \rightarrow \mathbb{R}$ such that $X$ is $\mathcal{A}$-measurable and bounded $\}$ ) such that $g$ is positive and:

$$
(\mathcal{S})\left\{\begin{array}{l}
g\left(\Omega^{*}\right) \geqslant v(\Omega) \\
g\left(-\Omega^{*}\right) \geqslant-v(\Omega) \\
g\left(-A^{*}\right) \geqslant-v(A) ; \forall A \in \mathcal{A} .
\end{array}\right.
$$

Let $\beta_{1}, \beta_{2}, \ldots, \beta_{n}>0$ and $\Omega^{*},-\Omega^{*}, A_{j}^{*} \in \mathcal{V}\left(\right.$ for $\left.3 \leqslant j \leqslant n ; A_{j} \in \mathcal{A}\right)$ such that:

$$
\left\|\beta_{1} \Omega^{*}+\beta_{2}\left(-\Omega^{*}\right)+\sum_{j=3}^{n} \beta_{j}\left(-A_{j}^{*}\right)\right\|_{\infty}=1 .
$$

Then $\beta_{1} \Omega^{*}+\beta_{2}\left(-\Omega^{*}\right)+\sum_{j=3}^{n} \beta_{j}\left(-A_{j}^{*}\right) \leqslant \Omega^{*}$, so $\beta_{1} \Omega^{*} \leqslant \Omega^{*}+\beta_{2} \Omega^{*}+\sum_{j=3}^{n} \beta_{j} A_{j}^{*}$ thus $\beta_{1} v(\Omega) \leqslant v(\Omega)+\beta_{2} v(\Omega)+\sum_{j=3}^{n} \beta_{j} v\left(A_{j}\right)$, hence

$$
\beta_{1} v(\Omega)+\beta_{2}(-v(\Omega))+\sum_{j=3}^{n} \beta_{j}\left(-v\left(A_{j}\right)\right) \leqslant v(\Omega)<\infty .
$$

Corollary 2.3. $v$ is totally anti-balanced iff: $\forall n \in \mathbb{N} ; \forall a_{1}, \ldots, a_{n} \geqslant 0, \forall A \in \mathcal{A}$ and $\forall A_{1}, \ldots, A_{n} \in \mathcal{A} \cap A$, the following implication holds: $\sum_{i} a_{i} A_{i}^{*} \geqslant A^{*} \Rightarrow \sum_{i} a_{i} v\left(A_{i}\right) \geqslant v(A)$. 


\section{Distorted Probabilities}

Let $f:[0,1] \rightarrow[0,1]$ with $f(0)=0$ and $f(1)=1 . f$ is said to be star-shaped at $t \in[0,1]$, if $x \mapsto \frac{f(t)-f(x)}{t-x}$ is nondecreasing on $[0,1] \backslash\{t\}$. In the following remark, we present some characterizations of star-shaped functions which will be useful thereafter, most of these characterizations rise from definitions by simple calculations. We give a proof of (iv) which appears to be more relevant.

Remark 3.1. (i) $f$ is star-shaped at 0 if and only if $\frac{f(x)}{x} \leqslant \frac{f(y)-f(x)}{y-x}, \forall 0<x<y \leqslant 1$.

(ii) $f$ is star-shaped at 1 if and only if $\frac{1-f(y)}{1-y} \geqslant \frac{f(y)-f(x)}{y-x}, \forall 0 \leqslant x<y<1$.

(iii) $f$ is star-shaped at 1 if and only if $\frac{\bar{f}(x)}{x} \geqslant \frac{\bar{f}(y)}{y}, \forall 0<x \leqslant y \leqslant 1$. Where $\bar{f}()=$. $1-f(1-$.$) is the conjugate function of f$.

(iv) $f$ is star-shaped at 0 and 1 if and only if $f=\min _{i \in I} f_{i}$ where $I$ is a non-empty set and $f_{i}:[0,1] \rightarrow[0,1]$ is convex satisfying $f_{i}(0)=0$ and $f_{i}(1)=1, \forall i \in I$.

Proof. While the minimum of concave functions is a concave function, the minimum of convex functions is a star-shaped function.

Necessary Condition Let $\mathcal{G}=\{g:[0,1] \rightarrow[0,1] \mid g(0)=0, g(1)=1, g$ is convex and $g \geqslant f\}$. Then $\mathcal{G} \neq \emptyset$ because $i d \in \mathcal{G}$. We prove $f=\min _{g \in \mathcal{G}} g$ i.e $\forall x \in[0,1]: f(x)=$ $\min _{g \in \mathcal{G}} g(x)$. Let $x \in(0,1)$, and $g_{x}:[0,1] \rightarrow[0,1]$ defined by

$$
g_{x}(t)=\left\{\begin{array}{l}
\frac{f(x)}{x} t \text { on }(0, x] ; g_{x}(0)=0 \\
\frac{1-f(x)}{1-x} t+1-\frac{1-f(x)}{1-x} \text { on }[x, 1) ; g_{x}(1)=1
\end{array}\right.
$$

then $g_{x}$ is convex (because $\frac{f(x)}{x} \leqslant \frac{1-f(x)}{1-x}$ since $f$ is star-shaped at 0 and 1 ), $g_{x} \geqslant f$ and $g_{x}(x)=f(x)$.

Sufficient Condition Let $0<x<y \leqslant 1$ then $\exists j \in I / f(y)=f_{j}(y)$ hence

$$
\frac{f(x)}{x} \leqslant \frac{f_{j}(x)}{x} \leqslant \frac{f_{j}(y)-f_{j}(x)}{y-x} \leqslant \frac{f_{j}(y)-f(x)}{y-x}=\frac{f(y)-f(x)}{y-x}
$$

Then $f$ is star-shaped at 0 (proposition 3.1 (i)).

Let $0 \leqslant x<y<1$ then $\exists i \in I / f(x)=f_{i}(x)$ hence

$$
\frac{1-f(y)}{1-y} \geqslant \frac{1-f_{i}(y)}{1-y} \geqslant \frac{f_{i}(y)-f_{i}(x)}{y-x} \geqslant \frac{f(y)-f_{i}(x)}{y-x}=\frac{f(y)-f(x)}{y-x}
$$

Then $f$ is star-shaped at 1 . 
Points (i) and (ii) of the preceding remark 3.1 make it possible to give a geometrical interpretation of star-shapedness at 0 and 1 , indeed a function $f$ is star-shaped at 0 and 1 if and only if for any $x \in[0,1]$, the cord connecting the point with co-ordinates $(x, f(x))$ to the origin (to the point with co-ordinates $(1, f(1)=1$ ) respectively) is above the curve of $f$ on the interval $[0, x]$ (respectively $[x, 1]$ ).

Remark 3.2. (i) If $f$ is convex then $f$ is star-shaped at every point of $[0,1]$.

(ii) If $f$ is star-shaped at 0 and 1 then $f$ is nondecreasing on $[0,1]$ and continuous on $[0,1)$.

Proof. (ii) Monotonicity follows obviously from the fact that $f$ is star-shaped at 0 . Continuity arises from star-shapedness at 1 : The functions $f$ and $t \longmapsto \frac{1-f(t)}{1-t}$ are monotone, thus have limits on the right and on the left at any point of $(0,1)$, a limit on the right at 0 and one limit on the left at 1 . Let $x \in[0,1)$; we have: $\frac{1-f(x)}{1-x} \leqslant \frac{1-f\left(x^{+}\right)}{1-x}$ then $f\left(x^{+}\right) \leqslant f(x)$ but $f\left(x^{+}\right) \geqslant f(x)$ thus $f(x)=f\left(x^{+}\right)$. Let $x \in(0,1)$; we have: $\frac{1-f\left(x^{-}\right)}{1-x} \leqslant \frac{1-f(x)}{1-x}$ then $f\left(x^{-}\right) \geqslant f(x)$ but $f\left(x^{-}\right) \leqslant f(x)$ thus $f(x)=f\left(x^{-}\right)$. Hence $f$ is continuous at $x ; \forall x \in(0,1)$ and $f$ is continuous on the right at 0 .

A probability $P$ on $(\Omega, \mathcal{A})$ is said to be non-atomic if $\forall A \in \mathcal{A}, \forall \alpha \in[0, P(A)]: \exists B \in$ $\mathcal{A}$ and $B \subset A \mid P(B)=\alpha$.

In the sequel of this section $\mathcal{A}$ is a $\sigma$-algebra of subsets of $\Omega, P$ is a non-atomic probability on $(\Omega, \mathcal{A})$ and $f:[0,1] \rightarrow[0,1]$ is a nondecreasing function satisfying $f(0)=0$ and $f(1)=1$. Denote by $v$ the capacity $v=f \circ P$. In some economic applications, $P$ represents the initial distribution of resources and $f$ represents a production function.

In the following proposition, we give a necessary and sufficient condition on the transformation $f$ of the probability so that capacity $v$ is balanced.

Proposition 3.1. $v$ is balanced if and only if $f(x) \leqslant x ; \forall x \in[0,1]$.

Proof. Assume $v$ is balanced. We show $f\left(\frac{m}{n}\right) \leqslant \frac{m}{n} ; \forall n \in \mathbb{N}^{*}, \forall m \in\{1 \ldots, n\}$. Let $n \in \mathbb{N}^{*}$, since $\mathrm{P}$ is non-atomic, $\exists A_{1} \ldots, A_{n} \in \mathcal{A}$ such that $P\left(A_{i}\right)=\frac{1}{n} \forall i \in\{1 \ldots, n\}$ and $A_{i} \cap A_{j}=$ $\emptyset \forall i, j \in\{1 \ldots, n\}$ with $i \neq j$. Let $m \in\{1 \ldots, n\}$; denote $\mathcal{C}=\{I \subset\{1, \ldots, n\} /|I|=m\}$, the cardinal of $\mathcal{C}$ is then $|\mathcal{C}|=C_{n}^{m}=\frac{n !}{m !(n-m) !}$. For $I \in \mathcal{C}$ : let $A_{I}=\cup_{i \in I} A_{i}$ (thus $A_{I} \in \mathcal{A}$ and $P\left(A_{I}\right)=\frac{m}{n}$ ) then: $C_{n-1}^{m-1} \Omega^{*}=\sum_{I \in \mathcal{C}} A_{I}^{*}$ (because for $i$ fixed in $\{1, \ldots, n\}$; the number of subsets $I \in \mathcal{C}$ and containing $i$ is $\left.C_{n-1}^{m-1}\right)$. Since $v$ is balanced, we have: $C_{n-1}^{m-1} \geqslant \sum_{I \in \mathcal{C}} v\left(A_{I}\right)=\sum_{I \in \mathcal{C}} f\left(\frac{m}{n}\right)=C_{n}^{m} f\left(\frac{m}{n}\right)$ that is $f\left(\frac{m}{n}\right) \leqslant \frac{C_{n-1}^{m-1}}{C_{n}^{m}}=\frac{m}{n}$. Therefore $\forall r \in \mathbb{Q} \cap[0,1]: f(r) \leqslant r$. We show then (knowing that $\mathrm{f}$ is nondecreasing) that $f(x) \leqslant$ $x ; \forall x \in[0,1)$. Let $x \in[0,1)$ and $\left(r_{n}\right)_{n \in \mathbb{N}}$ a sequence of rationals which decreases towards $x$ then $\forall n: f\left(r_{n}\right) \leqslant r_{n}$ thus, taking the limits limits, we get $f\left(x^{+}\right) \leqslant x$ but $f\left(x^{+}\right) \geqslant f(x)$ hence $f(x) \leqslant x ; \forall x \in[0,1)$ and the inequality is clearly satisfied at 1 .

The converse is straightforward since $P \in \operatorname{core}(v)$, thus $v$ is balanced. 
The following proposition characterizes totally balanced capacities $v=f \circ P$.

Proposition 3.2. $v$ is totally balanced if and only if $f$ is star-shaped at 0 .

Proof. Assume that $v$ is totally balanced. To show that $f$ is star-shaped at 0 , it is enough to prove: for all $n \in \mathbb{N}^{*}$ and $1<m \leqslant n: \frac{f\left(\frac{m}{n}\right)}{\frac{m}{n}} \geqslant \frac{f\left(\frac{m-1}{n}\right)}{\frac{m-1}{n}}$. Let $n \in \mathbb{N}^{*}$ : since $\mathrm{P}$ is non-atomic, $\exists A_{1} \ldots, A_{n} \in \mathcal{A}$ such that $P\left(A_{i}\right)=\frac{1}{n} \forall i \in\{1 \ldots, n\}$ and $A_{i} \cap A_{j}=\emptyset \forall i, j \in\{1 \ldots, n\}$ with $i \neq j$. Let $A=\cup_{j=1}^{m} A_{j}$ where $1<m \leqslant n$. Then: $(m-1) A^{*}=\sum_{k=1}^{m}\left(\cup_{j=1_{j \neq k}}^{m} A_{j}\right)^{*}$, hence: $(m-1) v(A) \geqslant \sum_{k=1}^{m} v\left(\cup_{j=1_{j \neq k}}^{m} A_{j}\right)$ i.e: $(m-1) f\left(\frac{m}{n}\right) \geqslant \sum_{k=1}^{m} f\left(\frac{m-1}{n}\right)=m f\left(\frac{m-1}{n}\right)$ Thus: $\frac{f\left(\frac{m}{n}\right)}{\frac{m}{n}} \geqslant \frac{f\left(\frac{m-1}{n}\right)}{\frac{m-1}{n}}$. That is: $\frac{f(a)}{a} \leqslant \frac{f(b)}{b} \forall a, b \in \mathbb{Q} \cap(0,1]$ with $a \leqslant b$, and $f$ being monotone (nondecreasing), has a limit on the left and on the right at any point $x \in(0,1)$, a limit on the right at 0 and a limit on the left at 1 . Let $x, y \in(0,1] \mid x<y ;\left(a_{n}\right)_{n \in \mathbb{N}}$ and $\left(b_{n}\right)_{n \in \mathbb{N}}$ two sequences of rationals in $(0,1]$ such that $a_{n}$ decreases towards $x$ and $b_{n}$ increases towards $y$ (then for $n$ large enough: $a_{n} \leqslant b_{n}$ ), thus for $n$ large enough: $\frac{f\left(a_{n}\right)}{a_{n}} \leqslant \frac{f\left(b_{n}\right)}{b_{n}}$. Taking the limits when $n \rightarrow \infty$, we obtain: $\frac{f\left(x^{+}\right)}{x} \leqslant \frac{f\left(y^{-}\right)}{y}$ however $f\left(x^{+}\right) \geqslant f(x)$ and $f\left(y^{-}\right) \leqslant f(y)$ (because $f$ is nondecreasing) hence $\frac{f(x)}{x} \leqslant \frac{f(y)}{y}$ and then: $f$ is star-shaped at 0 .

Conversely, assume that $f$ is star-shaped at 0 and let $A \in \mathcal{A}$. We show that $\operatorname{core}\left(v_{\mid A}\right) \neq$ $\emptyset$ i.e there exists $\lambda: \mathcal{A} \cap A \rightarrow[0, \infty]$ additive s.t $\lambda(A)=v_{\mid A}(A)=v(A)$ and $\lambda \geqslant v_{\mid A}$ (i.e. $\left.\lambda(B) \geqslant v_{\mid A}(B)=v(B), \forall B \in \mathcal{A} \cap A\right)$. Since $f$ is star-shaped at 0 , we get $\forall B \in \mathcal{A} \cap A$ : $v(B) \leqslant P(B) \frac{v(A)}{P(A)}$ then $\lambda=\frac{v(A)}{P(A)} P$ is appropriate, under the convention $\frac{0}{0}=1$.

Star-shapedness of $f$ at 1 characterizes the total anti-balancedness of $\bar{v}$.

Proposition 3.3. $f$ is star-shaped at 1 iff $\bar{v}$ is totally anti-balanced.

Proof. The proof is analogous to the proof of the previous proposition. Assume $\bar{v}$ is totally anti-balanced, to show that $f$ is star-shaped at 1 , it is enough to prove that: $\forall n \in \mathbb{N}^{*}, \forall 0 \leqslant$ $m<n-1$ we have: $\frac{1-f\left(\frac{m}{n}\right)}{1-\frac{m}{m}} \leqslant \frac{1-f\left(\frac{m+1}{n}\right)}{1-\frac{m+1}{n}}$. Let $n \in \mathbb{N}^{*},\left(A_{i}\right)_{1 \leqslant i \leqslant n}$ a partition of $\Omega$ composed by elements of $\mathcal{A}$ such that $P\left(A_{i}\right)=\frac{1}{n} \forall i \in\{1 \ldots, n\}$. Let $A=\cup_{j=1}^{n-m} A_{j}$ where $0 \leqslant m<n-1$, then using proposition 2.1, we get the desired result.

Conversely, assume $f$ is star-shaped at 1 and let $A \in \mathcal{A}$. Then, under the usual convention $\frac{0}{0}=1, \lambda()=.\frac{1-v(\bar{A})}{1-P(\bar{A})} P($.$) is in anti -\operatorname{core}\left(\bar{v}_{\mid A}\right)$.

Now we characterize capacities $v=f \circ P$ which are exact.

Proposition 3.4. $v$ is exact if and only if $f$ is star-shaped at 0 and 1 .

Proof. If $v$ is exact then $v$ is totally balanced and $\bar{v}$ is totally anti-balanced. Hence $f$ is star-shaped at 0 and 1.

If $f$ is star-shaped at 0 and 1 then we have $f=\min _{i \in I} f_{i}$ where $I \neq \emptyset$ and the $f_{i}$ 's are convex satisfying $f_{i}(0)=0$ and $f_{i}(1)=1$. Thus $v=f \circ P=\min _{i \in I} v_{i}$, where $v_{i}=f_{i} \circ P$ 
are convex capacities (since $f_{i}$ 's are, according to proposition 3.5 below) therefore exact. Let $A \in \mathcal{A}: \exists i \in I \mid v(A)=v_{i}(A)$ since $v_{i}$ is exact, $\exists \lambda_{i} \in \operatorname{core}\left(v_{i}\right) \mid \lambda_{i}(A)=v_{i}(A)=v(A)$ but $\operatorname{core}\left(v_{i}\right) \subset \operatorname{core}(v)$ because $v_{i} \geqslant v$ thus $\lambda_{i} \in \operatorname{core}(v)$ and $\lambda_{i}(A)=v(A)$.

The following result appears in [1].

Proposition 3.5. $v$ is convex if and only if $f$ is convex.

Proof. Assume $v$ is convex. Let $a, b \in[0,1]$ and consider $A, B \in \mathcal{A}$ such that $P(A \cup B)=a$ and $P(A \cap B)=b$ (this is possible since $P$ is without atoms). Then $v(A \cup B)+v(A \cap B) \geqslant$ $v(A)+v(B)$ can be written $f(a)+f(b) \geqslant 2 f\left(\frac{a+b}{2}\right)$ hence $f$ is convex (since it is continuous).

Now, if $f$ is convex: since a probability is an increasing set function, we have

$$
\begin{aligned}
& P(A)=\alpha P(A \cap B)+(1-\alpha) P(A \cup B), \text { for some } \alpha \in[0,1] \\
& P(B)=\beta P(A \cap B)+(1-\beta) P(A \cup B), \text { for some } \beta \in[0,1]
\end{aligned}
$$

but $\frac{P(A)+P(B)}{2}=\frac{P(A \cup B)+P(A \cap B)}{2}$ thus $\alpha+\beta=1$ then

$$
\begin{aligned}
v(A)+v(B) & =f(P(A))+f(P(B)) \\
\leqslant & \alpha v(A \cap B)+(1-\alpha) v(A \cup B)+\alpha v(A \cup B)+(1-\alpha) v(A \cap B) \\
& =v(A \cap B)+v(A \cup B)
\end{aligned}
$$

i.e: $v$ is convex.

The following proposition is inspired by Wallner [13]. We give the explicit expression, in the case of a convex capacity $v=f \circ P$, of a probability in the core of $v$ which coincides with $v$ on a given finite chain of elements of the $\sigma$-algebra $\mathcal{A}$.

Proposition 3.6. If $f$ is convex and $\emptyset=A_{0} \subseteq A_{1} \subseteq \ldots \subseteq A_{n}=\Omega$ is a finite chain in $\mathcal{A}$ then, the probability defined (with convention $\frac{0}{0}=1$ ) by:

$$
\lambda(.)=\sum_{j=1}^{n}\left[v\left(A_{j}\right)-v\left(A_{j-1}\right)\right] P\left(. \mid A_{j} \backslash A_{j-1}\right)=\sum_{j=1}^{n} \frac{v\left(A_{j}\right)-v\left(A_{j-1}\right)}{P\left(A_{j}\right)-P\left(A_{j-1}\right)} P\left[\left(A_{j} \backslash A_{j-1}\right) \cap .\right]
$$

is in $\operatorname{core}(v)$ and satisfies: $\lambda\left(A_{j}\right)=v\left(A_{j}\right), \forall j \in\{0 \ldots, n\}$.

Proof. It is clear that $\lambda$ is a probability and that it coincides with $v$ on the chain.

Let $B \in \mathcal{A}$; we have

$$
\lambda(B)=1-\lambda(\bar{B})=1-\sum_{j=1}^{n} \frac{v\left(A_{j}\right)-v\left(A_{j-1}\right)}{P\left(A_{j}\right)-P\left(A_{j-1}\right)}\left[P\left(A_{j} \cap \bar{B}\right)-P\left(A_{j-1} \cap \bar{B}\right)\right.
$$


But $P(A \cap \bar{B})=P(A \cup B)-P(B)$ thus

$$
\lambda(B)=1-\sum_{j=1}^{n} \frac{v\left(A_{j}\right)-v\left(A_{j-1}\right)}{P\left(A_{j}\right)-P\left(A_{j-1}\right)}\left[P\left(A_{j} \cup B\right)-P\left(A_{j-1} \cup B\right)\right]
$$

and since $f$ is convex

$$
\frac{v\left(A_{j}\right)-v\left(A_{j-1}\right)}{P\left(A_{j}\right)-P\left(A_{j-1}\right)}\left[P\left(A_{j} \cup B\right)-P\left(A_{j-1} \cup B\right)\right] \leqslant\left[v\left(A_{j} \cup B\right)-v\left(A_{j-1} \cup B\right)\right]
$$

then $\lambda(B) \geqslant 1-\sum_{j=1}^{n}\left[v\left(A_{j} \cup B\right)-v\left(A_{j-1} \cup B\right)\right]=v(B)$. That is $\lambda \in \operatorname{core}(v)$.

\section{Yaari's model, capacities and risk aversion}

Let $P$ be a non-atomic $\sigma$-additive probability on $(\Omega, \mathcal{A})$ and $\succeq$ a nontrivial weak order on $\mathcal{B}_{\infty}(\Omega, \mathcal{A})$, the set of measurable and bounded random variables on $\Omega$, satisfying:

- Continuity w.r.t. monotone convergence: $X_{n} \uparrow X, X_{n} \leqslant Y \Rightarrow Y \succeq X$ and $X_{n} \downarrow$ $X, X_{n} \geqslant Y \Rightarrow X \succeq Y$;

- Monotonicity: $\epsilon>0, X \geqslant Y+\epsilon \Omega^{*} \Rightarrow X \succ Y$;

- Co-monotonic independence: $X \sim Y, Z$ is co-monotonic with $X$ and $Y$ implies $X+Z \sim Y+Z$

- First order stochastic dominance: $A, B \in \mathcal{A}: P(A) \geqslant P(B) \Rightarrow A^{*} \succeq B^{*}$.

Under these assumptions one obtains Yaari's model, namely there exists a capacity $v$ s.t. $\succeq$ is represented by the functional:

$$
I(X)=\int X d v=\int_{-\infty}^{0}(v(X \geqslant t)-1) d t+\int_{0}^{+\infty} v(X \geqslant t) d t,
$$

and moreover, there exists a unique continuous non-decreasing mapping $f:[0,1] \rightarrow[0,1]$ such that $f(0)=0, f(1)=1$ and $v=f \circ P$. (See [2], [11] and [14] for further details).

We recall in the following, the definitions of four notions of mean-preserving increase in risk, introduced in [3].

Let $Z$ be a random variable. We denote by $F=F_{Z}$ the cumulative distribution function of $Z$ (for $x \in \mathbb{R}: F(x)=P(Z \leqslant x)$ ), $F^{-1}$ the generalized inverse of $F$, for $p \in[0,1): F^{-1}(p)=\inf \{x \in \mathbb{R} \mid F(x)>p\}$.

Let $X$ and $Y$ be two random variables having the same mean.

$Y$ is said to be a general mean preserving increase in risk (MPIR) of $X$ if $\frac{1}{p} \int_{0}^{p} F_{X}^{-1}(t) d t \geqslant$ $\frac{1}{p} \int_{0}^{p} F_{Y}^{-1}(t) d t ; \forall p \in[0,1]$. Which means that the expected position for the $p \%$ smallest values is greater for $X$ than for $Y$. 
$Y$ is a monotone mean preserving increase in risk (M-MPIR) of $X$ if: $F_{X}^{-1}(p)-$ $F_{Y}^{-1}(p)$ is a non-increasing function of $p \in(0,1)$. Which can be written: $F_{X}^{-1}(q)-F_{X}^{-1}(p) \leqslant$ $F_{Y}^{-1}(q)-F_{Y}^{-1}(p) ; \forall 0<p<q<1$. This means that the interquantile intervals are shorter for $X$ than for $Y$.

$Y$ is a left monotone mean preserving increase in risk (LM-MPIR) of $X$ if $\frac{1}{p} \int_{0}^{p}\left[F_{X}^{-1}(t)-\right.$ $\left.F_{Y}^{-1}(t)\right] d t$ is a non-increasing function of $p \in[0,1]$. The additional expected gain of $X$ upon $Y$ for the $p \%$ smallest gains is non-increasing in $p$.

$Y$ is a right monotone mean preserving increase in risk (RM-MPIR) of $X$ if $\frac{1}{1-p} \int_{p}^{1}\left[F_{X}^{-1}(t)-\right.$ $\left.F_{Y}^{-1}(t)\right] d t$ is a non-increasing function of $p \in[0,1]$. The additional expected gain of $X$ upon $Y$ for the $(1-p) \%$ highest gains is non-increasing in $p$.

To each concept of increase in risk corresponds a concept of risk aversion:

A decision maker (DM) is strongly risk averse (monotone risk averse, left monotone risk averse, right monotone risk averse, respectively) if for any random variables $X$ and $Y$ such that $Y$ is a MPIR (M-MPIR, LM-MPIR, RM-MPIR, respectively) of $X$, the decision maker prefers $X$ to $Y$.

Building upon Lemma 4 in [3], the following result is immediate.

Proposition 4.1. In Yaari's model

- The DM is right monotone risk averse if and only if $v$ is totally balanced.

- The DM is left monotone risk averse if and only if $\bar{v}$ is totally anti-balanced.

\section{Concluding Remarks}

In this paper we aimed at showing that for distorted probabilities, classical properties of capacities as balancedness, convexity, exactness, etc... prove to be related to simple properties of the distortion function, and moreover that these classical properties of the capacity are intimately related to meaningful risk aversion attitudes for decision makers à la Yaari. This suggests that under uncertainty it might be fruitful to investigate for the Choquet expected utility model (Schmeidler (1989), Gilboa (1987)) which kind of uncertainty aversion is linked for instance with the use of totally balanced or exact capacities, in the same way as it has already been done for convex or balanced capacities (see for example: Schmeidler (1989), Chateauneuf and Tallon (2002)).

\section{References}

[1] A. CHATEAUNEUF, On the use of capacities in modeling uncertainty aversion and risk aversion, Journal of Mathematical Economics. 20 (1991), 343-369.

[2] A. CHATEAUNEUF, Modeling attitudes towards uncertainty and risk through the use of Choquet integral, Annals of Operations Research. 52 (1994), 3-20. 
[3] A. CHATEAUNEUF, Michèle COHEN and Isaac MEILIJSON, Four notions of meanpreserving increase in risk, risk attitudes and applications to the rank-dependent expected utility model, Journal of Mathematical Economics. 40 (2004), 547-571.

[4] A. CHATEAUNEUF and J-M. TALLON, Diversification, convex preferences and nonempty core in the Choqquet expected utility model, Economic Theory. 19 (2002), 509-523.

[5] F. DELBAEN, Convex games and extreme points, Journal of Mathematical Analysis and Applications. 45 (1974), 210-233.

[6] K. FAN, On systems of linear inequalities, Annals of Mathematical Studies. 38 (1956), 99-156.

[7] I. GILBOA, Expexted utility theory with purely subjective non-additive probabilities, Journal of Mathematical Economics. 16 (1987), 65-88.

[8] I. GILBOA and E. LEHRER, The value of information - An Axiomatic approach, Journal of Mathematical Economics. 20 (1991), 443-459

[9] Y. KANNAI, Countably additive measures in cores of games, Journal of Mathematical Analysis and Applications. 27 (1969), 227-240.

[10] D. SCHMEIDLER, Cores of exact games, I, Journal of Mathematical Analysis and Applications. 40, 214-225 (1972).

[11] D. SCHMEIDLER, Subjective probability and expected utility without additivity, Econometrica. 57, 571-587 (1989).

[12] L. S. SHAPLEY, Cores of convex games, International Journal of Game Theory 1 (1971), 12-26.

[13] A. WALLNER, Bi-elastic neighborhood models, Ludwig-Maximilians-University Munich, Germany.

[14] M. E. YAAri, The Dual theory of choice under risk, Econometrica. 55 (1987), 95-115. 